\title{
Clinical and dermoscopic changes of acquired melanocytic nevi of patients treated with afamelanotide
}

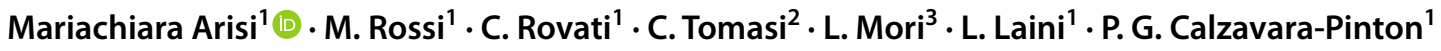

Received: 1 December 2020 / Accepted: 3 February 2021 / Published online: 17 February 2021

(c) The Author(s) 2021

\begin{abstract}
Background Afamelanotide (AFA) is a synthetic analogue of $\alpha$-melanocyte-stimulating hormone that is approved for the treatment of patients affected by erythropoietic protoporphyria (EPP). AFA induces a "sun free" tanning and changes of acquired melanocytic nevi (AMN) that are generically described as "darkening".

Objectives To assess clinical and dermoscopic AMN changes during AFA treatment.

Methods Adult EPP patients treated with two AFA implants 50 days apart were enrolled. They underwent a clinical and dermoscopic examination of all AMN at baseline (T0), and after 5 (T1) and 12 (T2) months from the first AFA implant. The general pattern, symmetry, number, and size of pigmented globules, morphology of the pigment network, and dermoscopic melanoma features were assessed.

Results Fifteen patients were enrolled with 103 AMN. At T1 all reticular and 2-component AMN showed a focal network thickening that returned to baseline by T2. The increase of globules' number was observed at T1 but not at T2. The difference in number was not influenced by patients' age or phototype. Dermoscopic changes suggestive of malignancy were never seen. The development of new AMN was never registered.

Conclusions AFA treatment induces reversible changes of AMN dermoscopic morphology without findings suggestive of malignant transformation and it does not stimulate the development of new AMN.
\end{abstract}

Keywords Afamelanotide $\cdot$ Melanocytic nevi $\cdot$ Dermoscopy $\cdot$ Melanoma

\section{Introduction}

The FECH gene encodes the synthesis of Ferrochelatase, a mitochondrial enzyme that catalyses the final step of the heme biosynthesis pathway, i.e. the insertion of ferrous iron into protoporphyrin IX (PpIX). Mutations in this gene lead to the build-up of metal-free PpIX in erythrocytes, plasma, skin and liver [1] that is the pathological substrate of the

Mariachiara Arisi

mariachiara.arisi@gmail.com

1 Dermatology Department, University of Brescia, ASST Spedali Civili Di Brescia, P.le Spedali Civili 1, 25123 Brescia, Italy

2 Department of Clinical and Experimental Sciences, University of Brescia, Brescia, Italy

3 Department of Clinical and Experimental Sciences, Molecular Medicine Laboratory, University of Brescia, Brescia, Italy clinical manifestations of erythropoietic protoporphyria (EPP).

As PpIX is photosensitive, the EPP patient develops episodes of burn and pain after even short solar exposures since early infancy $[2,3]$.

Until a few years ago, the cornerstone of the management of these patients was the absolute avoidance of sun exposures because the medical interventions, i.e. $\beta$-carotene, Vitamin C, cimetidine, $N$-acetyl-L-cysteine, and UVB phototherapy $[2,4,5]$, that were available at that time, had a poor preventative efficacy [6].

The therapeutic approach has been changed with the availability of Afamelanotide (Scenesse ${ }^{\circledR,}$ Clinuvel Pharmaceuticals, Melbourne, Australia) (AFA). It is a synthetic analogue of the human $\alpha$-melanocyte-stimulating hormone $(\alpha-\mathrm{MSH})$ that activates eumelanin synthesis by binding to the G-protein coupled melanocortin 1 receptor (MC1R) [1, 7] thus inducing a "sun free" tanning [8-10] that prevents PpIX photoactivation. Tan is more intense in subjects with a dark skin phototype [10], it increases after sun exposure and 
it fades after treatment discontinuation $[2,10]$. In 2 phase III trials, AFA was effective to prevent photosensitivity of EPP patients and the safety profile was good with only mild adverse effects, e.g. headache, nausea, nasopharyngitis, and back pain [1]. Changes, described generically as "darkening" of acquired melanocytic nevi (AMN) was also described [1] and this finding raised the theoretical concern that AFA can initiate or promote the growth of melanoma [2]. Therefore, the European Medical Agency (EMA) suggests precautionary a twice a year skin examination of all pigmentary lesions [11].

However, a careful clinical and dermoscopic investigation of AMN of AFA treated patients was never performed. This was the aim of the present study.

\section{Materials and methods}

\subsection{Patients}

Fifteen adult patients ( 8 males, 7 females) were enrolled and completed the study. All patients were referred to the Photobiology Unit, Dermatologic Department of the University of Brescia from January 2003 to December 2017. The age $($ mean $\pm \mathrm{SD})$ was $35.5 \pm 13.8$ years and 2,7 and 6 patients had skin phototype I, II, and III, respectively. Familial inheritance was found in six $(40 \%)$ patients. Nine $(60 \%)$ patients had the IVS3-48C polymorphism; other mutations reported in the $\mathrm{FECH}$ gene in our patients included c.252A > G, c.68-23C > T, c.901_902delTG, c.218_219insT, c.215_216insT, c. $-251 \mathrm{~A}>\mathrm{G}$.

All patients reported frequent phototoxic reactions after even short solar exposures. The reactions were erythematous in $9(60 \%)$ patients and/or oedematous in $11(73.3 \%)$ and were accompanied by itching in 7 patients $(46.6 \%)$ and/or burning and/or pain in 10 (66.6\%). Two patients had linear scars on the hands and face. The abdominal ultrasound showed cholelithiasis in one $(6.6 \%)$ case, previous cholecystectomy in 3 (20\%), liver steatosis in $2(13.3 \%)$ and hepatic angioma in $2(13.3 \%)$.

Patients received two AFA implants 50 days apart (February-April 2018) at the Photobiology Unit, Dermatologic Department of the University of Brescia. Patients were told to regularly apply spf50 + sunscreens and were restricted to sun exposure in the middle hours of the day (from $10 \mathrm{a}$.m. to 4 p.m.). At the other times, intentional sunbathing was discouraged but they could expose themselves during a normal relationship life, as far as individually tolerated. Women of childbearing age (5 patients) were on birth control therapy by consuming estroprogestinic pill and they repeated a pregnancy test before each implant.

The study was approved by Brescia Local Ethics Committee (Protocol Number 2688) and it was conducted in accordance with the Declaration of Helsinki. All patients were given verbal and written information on the nature of the study and they signed informed consent before enrolment.

\subsection{Study design}

Patients underwent a clinical and dermoscopic examination of all AMN of the whole skin surface at baseline (T0), and after 5 (T1) and 12 (T2) months from the first AFA implant. Lesions were classified into two groups on the basis of location in chronically light-exposed or non-light-exposed areas (gluteal and pubic areas in both sexes) during summertime. Total-body-photography and dermoscopic pictures of each pigmented lesion $\geq 4 \mathrm{~mm}$ in diameter were taken and stored [Vidix Vivascope 7 Videodermatoscope (Skin Imaging Group, Las Vegas, NV, USA)] at each examination. The lesion size was measured with the DERMASCOPE7 software (Skin Imaging Group, Las Vegas, NV, USA). We recorded the following dermoscopic features of each individual AMN: general pattern [globular, reticular, 2-component (combined globular plus reticular)]; lesion symmetry; number of pigmented globules; morphology of the pigment network of reticular and 2-component nevi $[12,13]$. In addition, we looked for dermoscopic features indicative of malignancy: atypical pigment network, regression areas (gray-blue areas), atypical vascular pattern, radial streaks, blotches, irregular dots and globules [12]. Finally, we looked carefully for the eventual appearance of novel AMN.

Dermoscopic pictures were independently evaluated by two investigators (MR and CR). In case of discrepancy in the evaluation, a third experimenter (AM) reviewed the dermoscopic images.

\subsection{Statistical analysis}

Kolmogorov-Smirnov test was performed to test the normality of the sample.

Wilcoxon signed-rank test and Chi-square test were used to compare dermoscopic changes (globules' number and diameter and network thickening respectively) after treatment.

To evaluate how dermoscopic features changed according to skin phototype, age, and lesion site (sun-exposed or sun-protected skin) simple linear regression was applied accordingly.

All statistical tests were conducted using the 5\% threshold $(p \leq 0.05)$ to reject the null hypothesis with two-tailed tests. Statistical analyses were performed using STATA ${ }^{\text {TM }}$ software ver. 14.2 and IBM-SPSS ${ }^{\text {TM }}$ software ver. 25.01.

The authors had full access to and take full responsibility for the integrity of the data. 


\section{Results}

Patients had a total number of 102 AMN that were mainly $(67 \%)$ located in non-exposed skin areas. The individual mean number of AMN was $6.8 \pm 5.3$ and all patients had AMN with more than 1 dermoscopic pattern. Baseline lesions' number, location, dermoscopic pattern and size are reported in detail in Table 1.

Kolmogorov-Smirnov test showed that continuous data were not normally distributed $(p<0.05)$.

The individual and total AMN numbers remained unchanged because novel AMN did not develop during the treatment period and follow-up. The average maximal diameter at baseline was $5.9 \pm 1.8 \mathrm{~mm}$ without changes at both $\mathrm{T} 1$ and T2. Lesion symmetry never changed (Table 1 ). The development of other pigmentary lesions was never registered.

At $\mathrm{T} 1$ we clinically observed hyperpigmentation of all AMN. On dermoscopic examination, all reticular and 2-component AMN showed a focal network thickening (Fig. 1d, e). Globules' number significantly increased at T1 $(p<0.0001$; Table 1, Fig. 1a, b) in all globular and 2-component nevi. At baseline average number of globules was $12.8 \pm 8.5$ vs. $18.9 \pm 10.5$ at $\mathrm{T} 1(p<0.0001)$. The increase of number of globules at $\mathrm{T} 1$ compared to $\mathrm{T} 0\left(\Delta_{\mathrm{T} 1-\mathrm{T} 0}\right)$ was not influenced by age $\left(p=0.13 ; Y_{\Delta \text { globules }}=-0.1 X_{\text {age }}+9.02\right)$, photoype $\left(p=0.88 ; Y_{\Delta \text { globules }}=-0.13 X_{\text {phototype }}+6.38\right)$ or body site $\left(p=0.7 ; Y_{\Delta \text { globules }}=-0.58 X_{\text {body site }}+6.5\right)$ in the given sample.
After 1 year (T2) all AMN showed a reduction of hyperpigmentation compared to $\mathrm{T} 1$ and their macroscopic features returned similar to baseline clinical findings. On dermoscopic examination, the pigment network in all reticular and 2-component nevi returned to baseline features (Fig. 1f). At T2, a decrease in the mean number (Table 1; Fig. 1c) of globules was recorded compared to T1 $(13.5 \pm 8.6$ vs. $18.9 \pm 10.5 ; p<0.0001)$ in all lesions. Difference between $\mathrm{T} 2$ and $\mathrm{T} 1\left(\Delta_{\mathrm{T} 2-\mathrm{T} 1}\right)$ was not influenced by age $\left(p=0.17 ; Y_{\Delta \text { globules }}=0.91 X_{\text {age }}-7.97\right)$, phototype $(p=0.87$; $Y_{\Delta \text { globules }}=0.15 X$ phototype -5.7$)$ or body site $(p=0.48$; $Y_{\Delta \text { globules }}=1.06 X_{\text {body site }}-6.1$ ).

Dermoscopic features suggestive of malignancy [12] were never observed at baseline and follow-up examinations.

\section{Discussion}

Previous studies on afamelanotide in PPE reported a macroscopical and clinical change, that was generically described as "darkening", of both AMN and lentigos regardless of body site but these changes were not previously investigated in detail by dermoscopy $[2,8,10]$.

In the present investigation, AFA treatment did not induce the development of novel AMN or other pigmentary lesions. Macroscopic changes of preexisting AMN were consistent with previous findings $[2,8,10]$ and were characterized by homogeneous hyperpigmentation, which was reversible after discontinuation of the drug.

Table 1 Clinical features of nevi at baseline (T0), 3-month follow-up after the second implant (T1) and 1 year after the first implant (T2)

\begin{tabular}{|c|c|c|c|c|c|}
\hline & T0 & $\mathrm{T} 1$ & $\mathrm{~T} 2$ & $p$ (T0 vs. T1) & $p$ (T1 vs. T2) \\
\hline Total number of nevi $n(\%)$ & $102(100 \%)$ & $102(100 \%)$ & $102(100 \%)$ & - & - \\
\hline Number of nevi per patient (mean \pm SD) & $6.8 \pm 5.3$ & $6.8 \pm 5.3$ & $6.8 \pm 5.3$ & - & - \\
\hline \multicolumn{6}{|l|}{ Location of nevi $n(\%)$} \\
\hline Covered skin areas ${ }^{\mathrm{a}}$ & $69(67.6 \%)$ & $69(67.6 \%)$ & $69(67.6 \%)$ & - & - \\
\hline UV-exposed skin areas & $33(32.3 \%)$ & $33(32.3 \%)$ & $33(32.3 \%)$ & & \\
\hline Pattern $n(\%)$ & & & & - & - \\
\hline Globular & $12(11.8 \%)$ & $12(11.8 \%)$ & $12(11.8 \%)$ & & \\
\hline Reticular & $68(66.7 \%)$ & $68(66.7 \%)$ & $68(66.7 \%)$ & & \\
\hline 2-components ) & $22(21.5 \%)$ & $22(21.5 \%)$ & $22(21.5 \%)$ & & \\
\hline Maximal diameter $(\mathrm{mm}):($ mean $\pm \mathrm{SD})$ & $5.9 \pm 1.8$ & $5.9 \pm 1.8$ & $5.9 \pm 1.8$ & - & - \\
\hline \multicolumn{6}{|l|}{ Symmetry $n(\%)$} \\
\hline Yes & $59(57.8)$ & $59(57.8)$ & $59(57.8)$ & - & \\
\hline No & $43(42.2)$ & $43(42.2)$ & $43(42.2)$ & & - \\
\hline Network thickening in reticular and 2-component nevi N (\%) & $0(0)$ & $90(100)$ & $0(0)$ & - & - \\
\hline $\begin{array}{l}\text { Number of globules (mean } \pm \text { DS) in each globular and 2-com- } \\
\text { ponent nevus }\end{array}$ & $12.8 \pm .8 .5$ & $18.9 \pm 10.5$ & $13.5 \pm .8 .6$ & $<0.0001$ & $<0.0001$ \\
\hline
\end{tabular}

TO baseline, T1 5 months after first implant, $T 212$ months after first implant

${ }^{\mathrm{a}}$ Gluteal and pubic region 


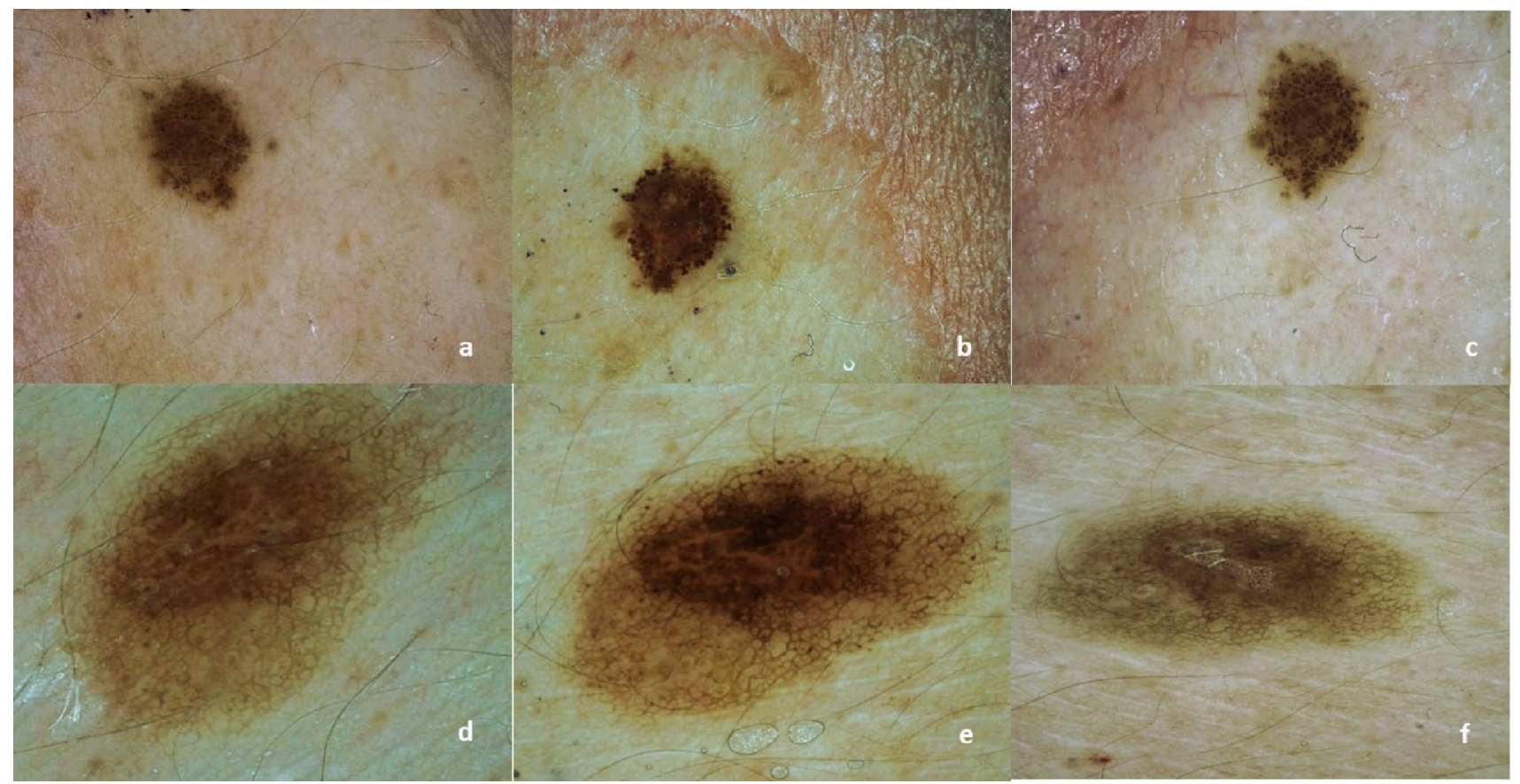

Fig. 1 Number of globules at different timepoints (10X): baseline (a), T1 (b) and 1-year follow-up (T2). Focal network thickening (red thick arrow) in 2-component nevi from baseline (d) to T2 (e). (20×) Following network normalization at 1-year follow-up (f) (10×)

Dermoscopically we did not observe changes of pigmentation homogeneity, size and symmetry of AMN and dermoscopic features that are characteristic of melanoma [12]. In our series, AFA treatment increased the number of globules in all globular and 2-component AMN and it induced the thickening of the pigment network in all reticular and 2-component lesions. These dermoscopic changes were seen in both sun exposed and sun-protected body sites after five months from the first implant (T1) and were not influenced by patients' age and skin phototypes. These dermoscopic clues were transient and after 1 year all AMN regained the same baseline morphology regardless of the body location.

Our results show AFA's ability to induce transient changes in the dermoscopic appearance of melanocytic nevi comparable to those induced by acute sun exposure [14] and artificial ultraviolet sources $[15,16]$. Some transient AFAinduced AMN changes also show overlaps to nevi modification during pregnancy [17]. In different case series of pregnant patients, dermoscopic features of melanocytic nevi have been assessed reporting thickening in pigment network $[18,19]$ or increased number of globules [20]. Similarly to AFA treatment, these pregnancy changes were reversible and not suggestive of melanoma.

Generally, the dermoscopic finding globules reflects the nests of the nevus cells in the dermis.

Given the reversibility of dermoscopic changes induced in a few weeks by the AFA in our series, it seems unlikely that the drug could activate pathways of cell proliferation on nevus nests. We hypothesize that the dermoscopic changes that we have observed are related only to effect on the synthesis of new melanin and that the transient increase in globules has been an expression of pigment removal blotches by macrophages.

Albeit, these results could be affected by the limited number of patients enrolled but they could also support the ability of AFA to induce AMN changes independently from constitutive pigmentogenic activity. Furthermore, another limitation of the present study was not to have ascertained AMN modifications by spectrophotometric evaluation or histology. Moreover, the present study did not investigate the effects of AFA on a pre-existing melanoma or precancerous lesion.

In our series, we never observed changes of size and symmetry, dermoscopic findings that are characteristic of melanoma [12] and novel melanocytic lesions. Altogether the present findings suggest that AFA treatment induces physiological and transient changes of AMN morphology without any findings that could indicate a carcinogenic activity.

The carcinogenic potential of the drug remains therefore only theoretical and based on contradictory evidences. Melanoma in AFA treated patients was never reported and also in phase 3 studies generic darkening of nevi has been described without the appearance of suspicious lesions [1].

Although the melanoma inducing potential of afamelanotide is still under debate, self-injection of unlicensed melanotan-II, a cyclic heptapeptide $\alpha$-MSH analogue that also 
binds to MC3R and MC4R, was recently associated with the development of multiple melanomas in a combined highrisk MC1R/CDKN2A genotype patient [21]. However, as the binding affinity of AFA for MC3R and MC4R are much lower than that for the MC1R [3], it is questionable as to whether they are activated by the pharmacological application of the drug itself [22].

Previous studies highlighted that serum $\alpha \mathrm{MSH}$ level was proportional to Breslow index and the titre was still higher in patients with metastatic melanoma [23, 24]. However, recent data have shown that $\alpha \mathrm{MSH}$ and MC1R have instead an anti-carcinogenic activity because not only they stimulate the production of eumelanin but also inhibit pro-inflammatory cytokines and reduce the expression of vascular-cell adhesion molecule (VCAM) and E-selectin [25]. In addition, $\mathrm{MC1R}$ is expressed by mature melanocytes but not by melanocyte stem cells or melanoblasts in melanocytic lesions [26]. Given the parallel with benign nevi, it is plausible to suppose that M1CR is variably expressed in melanoma and the more undifferentiated and therefore aggressive the melanoma is, the less the receptor is expressed in it [3]. Thus, the activation of the receptor itself, also by AFA, might induce a greater cellular differentiation rather than the opposite [27]. Moreover, increased $\alpha \mathrm{MSH}$ levels detected in melanoma patients may be associated with direct production by cancer melanocyte cells that have maintained reminiscence of their differentiated functions.

Another possible preventative role could be played by the high content of eumelanin in AFA-induced tan that could be particularly relevant for patients with fair phototypes whose natural tan has a high content of pro-carcinogenic pheomelanin.

In conclusion, AFA-induced MC1R stimulation of AMN melanocytes seems to induce only transient physiological changes of AMN morphology and as such, they do not support the concerns of carcinogenic activity of AFA. However, the role of AFA in promoting the growth of a melanoma already present remains unaddressed, a careful dermoscopic evaluation before the AFA treatment should be mandatory and long-term safety of AFA treatment has to be further investigated.

Funding Open access funding provided by Università degli Studi di Brescia within the CRUI-CARE Agreement.

\section{Compliance with ethical standard}

Conflict of interest The authors declare that they have no conflict of interest.

Open Access This article is licensed under a Creative Commons Attribution 4.0 International License, which permits use, sharing, adaptation, distribution and reproduction in any medium or format, as long as you give appropriate credit to the original author(s) and the source, provide a link to the Creative Commons licence, and indicate if changes were made. The images or other third party material in this article are included in the article's Creative Commons licence, unless indicated otherwise in a credit line to the material. If material is not included in the article's Creative Commons licence and your intended use is not permitted by statutory regulation or exceeds the permitted use, you will need to obtain permission directly from the copyright holder. To view a copy of this licence, visit http://creativecommons.org/licenses/by/4.0/.

\section{References}

1. Todd, D. J. (1994). Erythropoietic protoporphyria. British Journal of Dermatology, 131(6), 751-766.

2. Langendonk, J. G., Balwani, M., Anderson, K. E., Bonkovsky, H. L., Anstey, A. V., Bissell, D. M., et al. (2015). Afamelanotide for erythropoietic protoporphyria. The New England Journal of Medicine, 373(1), 48-59.

3. Minder, E. I. (2010). Afamelanotide, an agonistic analog of $\alpha$-melanocyte-stimulating hormone, in dermal phototoxicity of erythropoietic protoporphyria. Expert Opinion on Investigational Drugs, 19(12), 1591-1602.

4. Mathews-Roth, M. M., Pathak, U. A., Fitzpatrick, T. B., Harber, L. C., \& Kass, E. H. (1974). Beta-carotene as an oral photoprotective agent in erythropoietic protoporphyria. Journal of American Medical Association, 228(8), 1004-1008.

5. Tu, J. H., Sheu, S. L., \& Teng, J. M. (2016). Novel treatment using cimetidine for erythropoietic protoporphyria in children. JAMA Dermatology, 152(11), 1258-1261.

6. Minder, E. I., Schneider-Yin, X., Steurer, J., \& Bachmann, L. M. (2009). A systematic review of treatment options for dermal photosensitivity in erythropoietic protoporphyria. Cellular and Molecular Biology, 55(1), 84-97.

7. Fabrikant, J., Touloei, K., \& Brown, S. M. (2013). A review and update on melanocyte stimulating hormone therapy: afamelanotide. Journal of Drugs in Dermatology, 12(7), 775-779.

8. Harms, J. H., Lautenschlager, S., Minder, C. E., \& Minder, E. I. (2009). Mitigating photosensitivity of erythropoietic protoporphyria patients by an agonistic analog of alpha-melanocyte stimulating hormone. Photochemistry and Photobiology, 85(6), 1434-1439.

9. Biolcati, G., Deybach, J. C., \& Hanneken, S. (2011). A randomized Phase III trial of afamelanotide (Scenesse ${ }^{\circledR}$ ), an agonistic $\alpha$-melanocyte stimulating hormone analogue in the treatment of protoporphyria-induced phototoxicity. British Journal of Dermatology, 164(5), 1143.

10. Biolcati, G., Marchesini, E., Sorge, F., Barbieri, L., SchneiderYin, X., \& Minder, E. I. (2015). Long-term observational study of afamelanotide in 115 patients with erythropoietic protoporphyria. British Journal of Dermatology, 172(6), 1601-1612.

11. https://www.ema.europa.eu/en/documents/rmp-summary/scene sse-epar-risk-management-plan-summary_en.pdf. Accessed 27 May 2020

12. Argenziano, G., Fabbrocini, G., Carli, P., De Giorgi, V., Sammarco, E., \& Delfino, M. (1998). Epiluminescence microscopy for the diagnosis of doubtful melanocytic skin lesions. Comparison of the ABCD rule of dermatoscopy and a new 7-point checklist based on pattern analysis. Archives of Dermatology, 134(12), 1563-1570.

13. Rosendahl, C., Tschandl, P., Cameron, A., \& Kittler, H. (2011). Diagnostic accuracy of dermatoscopy for melanocytic and nonmelanocytic pigmented lesions. Journal of the American Academy of Dermatology, 64(6), 1068-1073.

14. Dobrosavljevic, D., Brasanac, D., Apostolovic, M., \& Medenica, L. (2009). Changes in common melanocytic naevi after intense 
sun exposure: digital dermoscopic study with a 1-year followup. Clinical and Experimental Dermatology, 34(6), 672-678.

15. Manganoni, A. M., Rossi, M. T., Sala, R., Venturini, M., Sereni, E., et al. (2012). Dermoscopic, histological and immunohistochemical evaluation of cancerous features in acquired melanocytic nevi that have been repeatedly exposed to UVA or UVB. Experimental Dermatology, 21(2), 86-90.

16. Kilinc Karaarslan, I., Teban, L., Dawid, M., Tanew, A., \& Kittler, H. (2007). Changes in the dermoscopic appearance of melanocytic naevi after photochemotherapy or narrow-band ultraviolet B phototherapy. Journal of the European Academy of Dermatology and Venereology, 21(4), 526-531.

17. Bieber, A. K., Martires, K. J., Driscoll, M. S., Grant-Kels, J. M., Pomeranz, M. K., \& Stein, J. A. (2016). Nevi and pregnancy. Journal of the American Academy of Dermatology, 75(4), 661-666.

18. Gunduz, K., Koltan, S., Sahin, M. T., \& Filiz, E. (2003). Analysis of melanocytic naevi by dermoscopy during pregnancy. Journal of the European Academy of Dermatology and Venereology, 17(3), 349-351.

19. Rubegni, P., Sbano, P., Burroni, M., Cevenini, G., Bocchi, C., Severi, F. M., et al. (2007). Melanocytic skin lesions and pregnancy: digital dermoscopy analysis. Skin Research and Technology, 13(2), 143-147.

20. Strumia, R. (2002). Digital epiluminescence microscopy in nevi during pregnancy. Dermatology, 205(2), 186-187.

21. Böhm, M., Jagirdar, K., Sturm, R. A., König, S., Bauer, J., Metze, D., et al. (2016). Lack of protection from development of multiple melanomas by an injected melanocortin analogue in a combined high-risk MC1R/CDKN2A genotype patient. Journal of the European Academy of Dermatology and Venereology, 30(10), e65-e67.

22. Minder, E. I., Barman-Aksoezen, J., \& Schneider-Yin, X. (2017). Pharmacokinetics and pharmacodynamics of afamelanotide and its clinical use in treating dermatologic disorders. Clinical Pharmacokinetics, 56(8), 815-823.

23. Ghanem, G., Verstegen, J., Libert, A., Arnould, R., \& Lejeune, F. (1989). Alpha-melanocyte-stimulating hormone immunoreactivity in human melanoma metastases extracts. Pigment Cell Research, 2(6), 519-523.

24. Ghanem, G., Verstegen, J., De Rijcke, S., Hanson, P., Van Onderbergen, A., Libert, A., et al. (1989). Studies on factors influencing human plasma alpha-MSH. Anticancer Research, 9(6), $1691-1696$

25. Luger, T. A., Brzoska, T., Scholzen, T. E., Kalden, D. H., Sunderkötter, C., Armstrong, C., et al. (2000). The role of alpha-MSH as a modulator of cutaneous inflammation. Annals of the New York Academy of Sciences, 917, 232-238.

26. Osawa, M., Egawa, G., Mak, S. S., Moriyama, M., Freter, R., Yonetani, S., et al. (2005). Molecular characterization of melanocyte stem cells in their niche. Development, 132(24), 5589-5599.

27. Jiang, J., Sharma, S. D., Nakamura, S., Lai, J. Y., Fink, J. L., Hruby, V. J., et al. (1995). The melanotropic peptide, [Nle4, D-Phe7] alpha-MSH, stimulates human melanoma tyrosinase activity and inhibits cell proliferation. Pigment Cell Research, $8(6), 314-323$. 